\title{
Lipoma of the corpus callosum
}

\author{
D. WALLACE 1 \\ From the Neurosurgery Department, Hospital for Sick Children, \\ Great Ormond Street, London
}

SYNOPSIS Lipoma of the corpus callosum is a rare congenital condition, often asymptomatic, but which may present as epilepsy, hemiplegia, dementia, or headaches. This paper reviews the condition and reports the only two cases which are known to the Hospital for Sick Children, Great Ormond Street, London. The second case demonstrated the value of computerised axial tomography (EMI scan) in making the diagnosis and showing associated anomalies.

Lipomas occur in most parts of the body, but intracranial lipomas are exceptionally rare. Lipoma of the corpus callosum was first described in 1856 by Rokitansky who discovered a case at necropsy. It was not until 1939 that the first diagnosis of the condition was made in life. The two cases reported below were diagnosed after radiological investigations. As is the case with other lipomas, lipoma of the corpus callosum may be asymptomatic or may produce a number of distressing symptoms. Unlike other lipomas, lipoma of the corpus callosum does not lend itself to surgical cure.

\section{CASE 1}

J.T., a girl born on 9 March 1944, was slow to pass her motor milestones. At 14 months she had a focal, right-sided seizure, with loss of consciousness, incontinence, and post-ictal right hemiplegia. She did not walk until 24 months and had a hemiplegic gait. At 2 years 3 months of age, there was a second fit. A diagnosis of pinealoma was made. Three years later she had an attack of status epilepticus lasting 48 hours, followed by coma for two days. On regaining consciousness, she was severely retarded and aphasic. The aphasia persisted. She resumed walking over the next three weeks. In 1949 she was reinvestigated and a diagnosis of 'tuberculoma of the brain, with calcification' was made. She was treated with anticonvulsants. In 1954, she was admitted to the Hospital for Sick Children for further study. She had been free from fits for six years.

\footnotetext{
1 Address for reprint requests: Mr D. Wallace, c/o Neurosurgical Unit, Royal Children's Hospital, Parkville, Melbourne, Victoria, Australia. (Accepted 12 July 1976.)
}

On examination in 1954 there was severe mental retardation. Speech was limited to six words. The fundi and visual fields were normal. There was mild right facial weakness and a right spastic hemiplegia. The plantar responses were equivocal. On skull radiography the left hemicranium was smaller than the right. The skull was small, and the vault thick. Calcification was present, deep in the frontal region, and the anteroposterior half-axial projections showed the typical configuration of a lipoma of the corpus callosum. Bilateral carotid angiography showed that the anterior cerebral arteries joined to form a common trunk which divided into callosomarginal and pericallosal arteries. The pericallosal arteries were large, tortuous, and passed straight through the lesion. The callosal vessels were displaced to the left of the midline, suggesting some degree of left hemiatrophy of the brain. A lumbar pneumoencephalogram showed uniform dilatation of the left lateral ventricle and widened sulci over that hemisphere. The right lateral ventricle was of normal size. The anterior horns and bodies of the lateral ventricle were displaced laterally by the lipoma. Agenesis of the splenium of the corpus callosum was noted.

Operative intervention was thought to be contraindicated. She was discharged home, and followed up regularly as an out-patient. She remains well though severely dysphasic, and attends a school for the handicapped, where she takes part in swimming and horseriding.

\section{CASE 2}

G.S., a $6 \frac{1}{2}$ year old boy, on 21 September 1974 suddenly complained of headache and nausea, and vomited twice. Several hours later he was found in a confused post-ictal state. He was admitted to hospital 
where he had a left-sided fit. He was given anticonvulsants. Next morning a dense left hemiplegia was noted and he was transferred to the Hospital for Sick Children.

On arrival he was drowsy, disorientated in time, with marked neck stiffness and a positive Kernig's test. There was left upper motor neurone facial weakness, and a flaccid left hemiparesis. Tendon reflexes were normal, and the plantar reflexes flexor. Next day he improved and a left homonymous hemianopia was demonstrable. Skull radiographs showed an oval shaped area of calcification enclosing an area of radiolucency in the anterior region of the corpus callosum, thought to be pathognomonic of a lipoma of the corpus callosum (Fig. 1). The left side of the skull vault was expanded and thinner than the right. The lipoma was slightly to the right of the midline and the crista galli was deviated to the right, consistent with the presence of right cerebral hemiatrophy. A right carotid angiogram with cross compression showed a single, grossly enlarged, anterior cerebral artery, with a curve of longer radius than usual (Fig. 2). It divided into two pericallosal and callosomarginal branches. The pericallosal vessels coursed through an avascular spacecccupying lesion in the region of the genu of the corpus callosum and posterior to it. The internal cerebral vein was abnormal in shape. The anterior portion appeared depressed and pushed backwards. In the venous phase the lateral ventricles were dilated. The EMI scan (Fig. 3) from the National Hospital, Queen Square (reported by Dr J. Gawler) showed the intracranial anatomy to be grossly abnormal. In the mid-frontal region there was a well-defined and smoothly marginated area, of density corresponding to fat. The lesion measured, at maximum, $4 \mathrm{~cm}$ in the anteroposterior plane, $3 \mathrm{~cm}$ in width, and was symmetrically disposed with respect to the midline. Posterosuperiorly, the lesion had paired processes which extended backwards in a symmetrical fashion, and these processes were separated by a thin band from paired symmetrical fatty cysts, each about $1 \mathrm{~cm}$ in diameter. The anterior aspect of the midline lesion was capped by a rim of calcification. The frontal horns and anterior parts of the bodies of the lateral ventricles could not be identified. On the right the trigone and occipital horn were dilated. On the left there was massive porencephalic enlargement of the ventricle, so that the trigone and occipital horn appeared to occupy the posterior half of the hemicranium. The left temporal horn was also quite dilated. The third ventricle, which extended backwards from the region of the fatty lesion, was dilated but lay in the midline. Posterior fossa scans showed a normal fourth ventricle. The skull was asymmetrical, the left hemicranium being larger than the right, and there was parieto-occipital bulging over the enormously dilated posterior segment of the left lateral ventricle.

He was treated with anticonvulsants and physiotherapy. His conscious state rapidly improved, and he became euphoric and excessively friendly. He had marked disorientation in time and space. Speech was normal. There was astereognosis and left-sided sensory inattention. The left homonymous hemianopia remained. There was a mild residual left facial weakness with mild left spastic hemiparesis. The tendon reflexes remained symmetrical and the plantar responses were flexor. He continued to improve and was discharged home on anticonvulsants. Out-patient reviews have revealed further improvement of the hemiparesis.

\section{DISCUSSION}

Lipoma of the corpus callosum, in one series, occurred only four times in 5000 necropsies (Vonderahe and Niemer, 1944). The first case discovered at necropsy at the Johns Hopkins Hospital occurred after 23813 consecutive necropsies (Patel, 1965). Thirty cases were reported by List and Everett (1946) (28 of these from the literature and two of their own). Fifteen additional cases were reported by Nordin et al. (1955). At the time of the survey by Zettner and Netsky in 1960 there were 59 cases reported in the literature of which $\mathbf{4 8}$ were diagnosed at necropsy or confirmed by surgical intervention. Patel (1965) reviewed 72 cases and reported one new case, Meyer and Leimbach (1970) collected a total of 70 cases recorded to 1969 . Since 1969 there have been : further sporadic case reports (Jayam et al., 1969; Bromowicz, 1970; Larsen and Stiris, 1970; Arseni and Maretsis, 1972; Craddock and Riddervold, 1972; Danziger, 1972; Sukthomya and Menakanit, 1973).

The consensus of opinion concerning the aetiology of lipoma of the corpus callosum is that it is a congenital, hamartomatous condition (Cooper and Von Hagen, 1962). It has been regarded as an example of 'cerebral dysraphism', the term dysraphism referring to the midline defects caused by imperfect closure of the neural tube. The dysraphic concept is supported by the occurrence in some of the reported cases of other dysraphic disturbances-for example, agenesis of the corpus callosum-occurring in $48 \%$ of cases (Zettner and Netsky, 1960), spina bifida and meningomyelocoele (Patel, 1965). However Zettner and Netsky (1960), in their most detailed account of normal and abnormal development of the corpus callosum, and the possible modes of origin of lipomas of the corpus callosum, pointed out that the commonest associated dysraphic disturbance, agenesis of the corpus callosum, occurs in only $48 \%$ of cases, and concluded that dysraphism was not the 


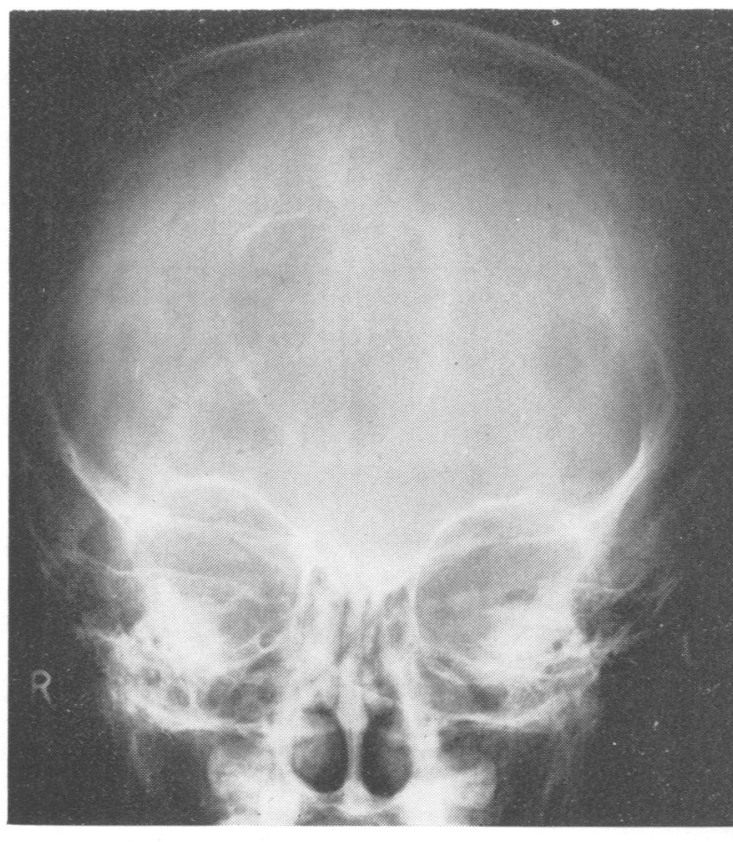

FIG. 1. Case 2. PA, AP, and lateral radiography of skull showing frontal radiolucent area with calcified rim.
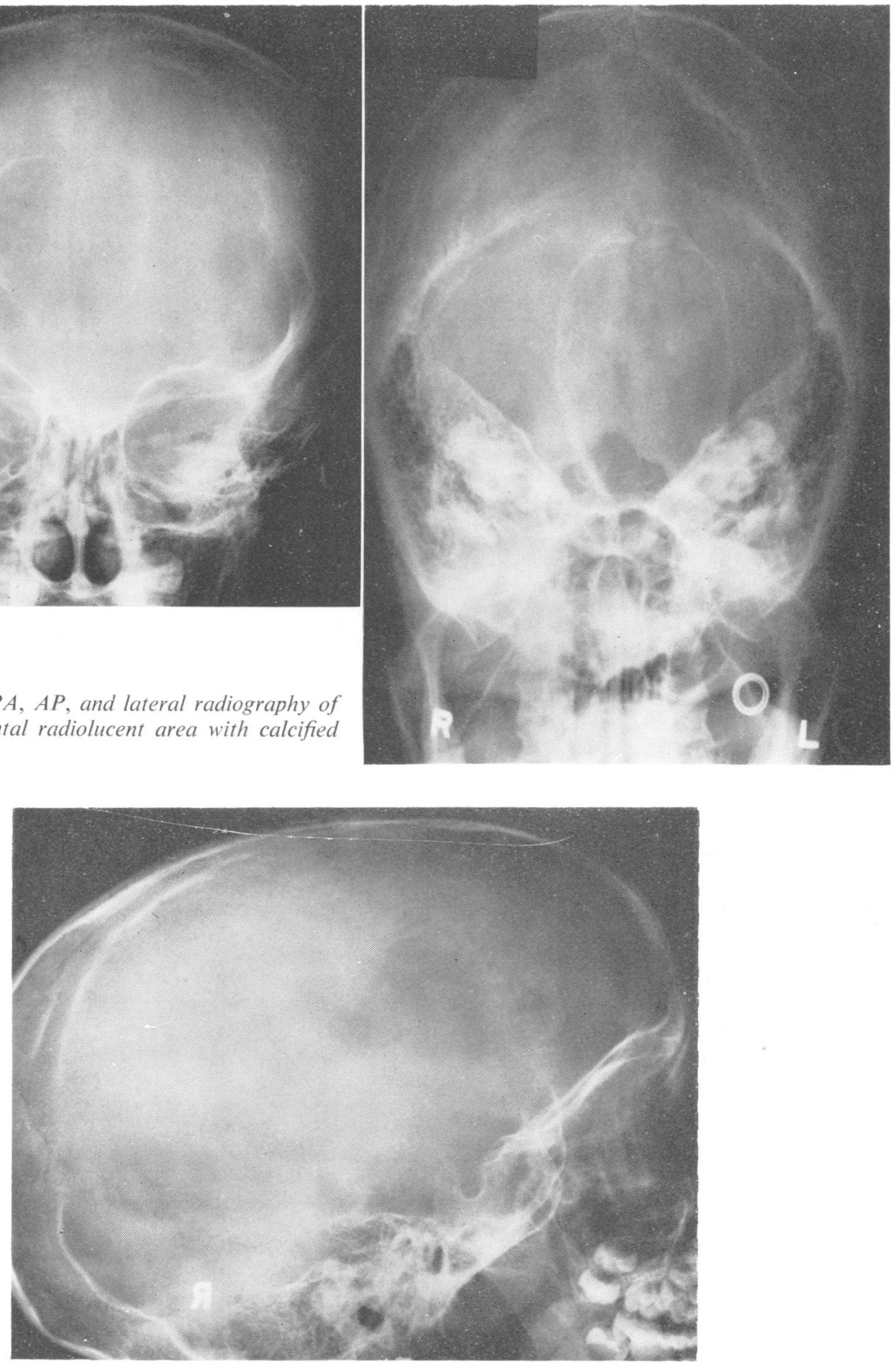

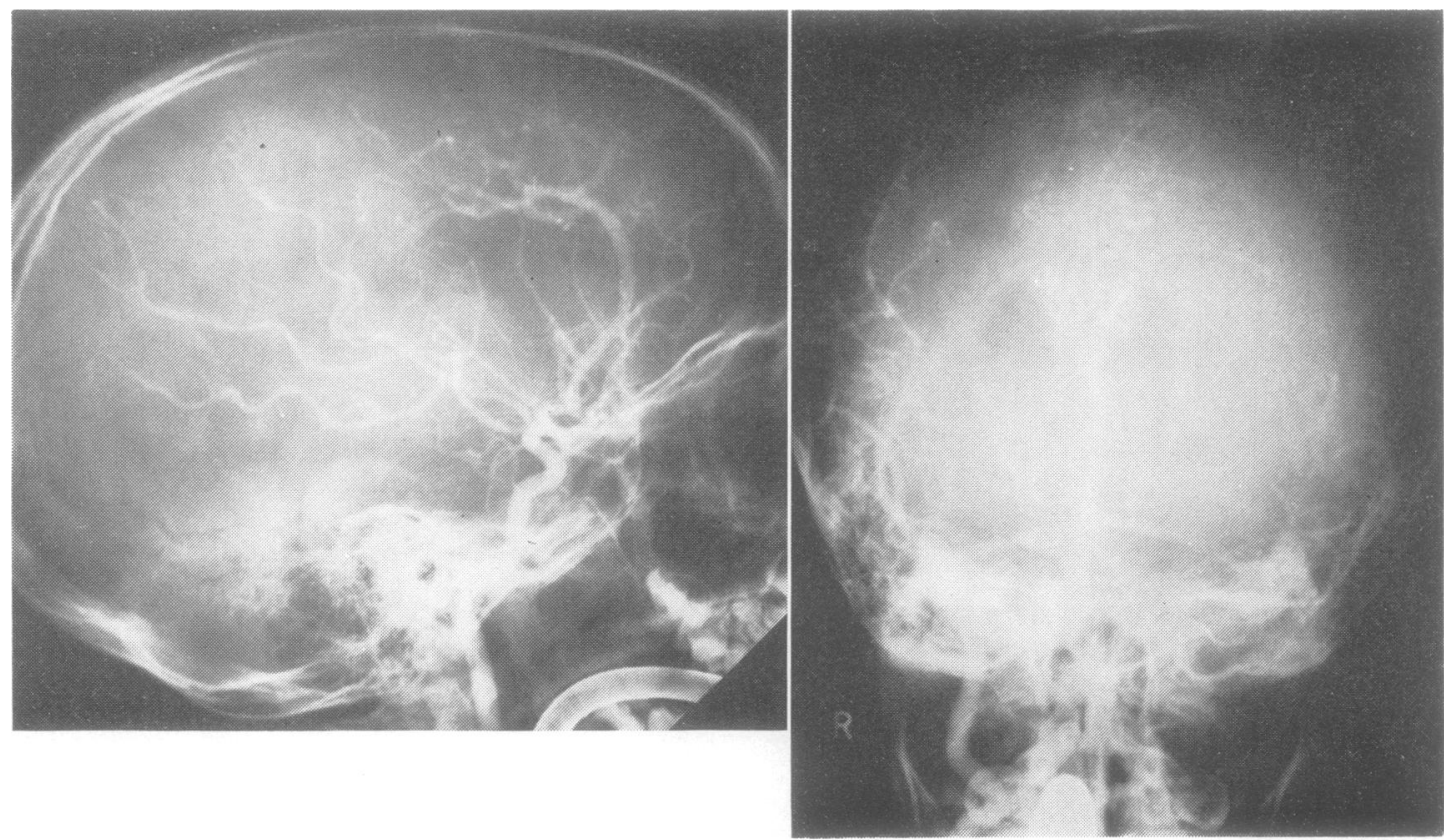

FIG. 2. Case 2. Lateral and AP carotid angiograms showing a large single, stretched, anterior cerebral artery passing through the lipoma.
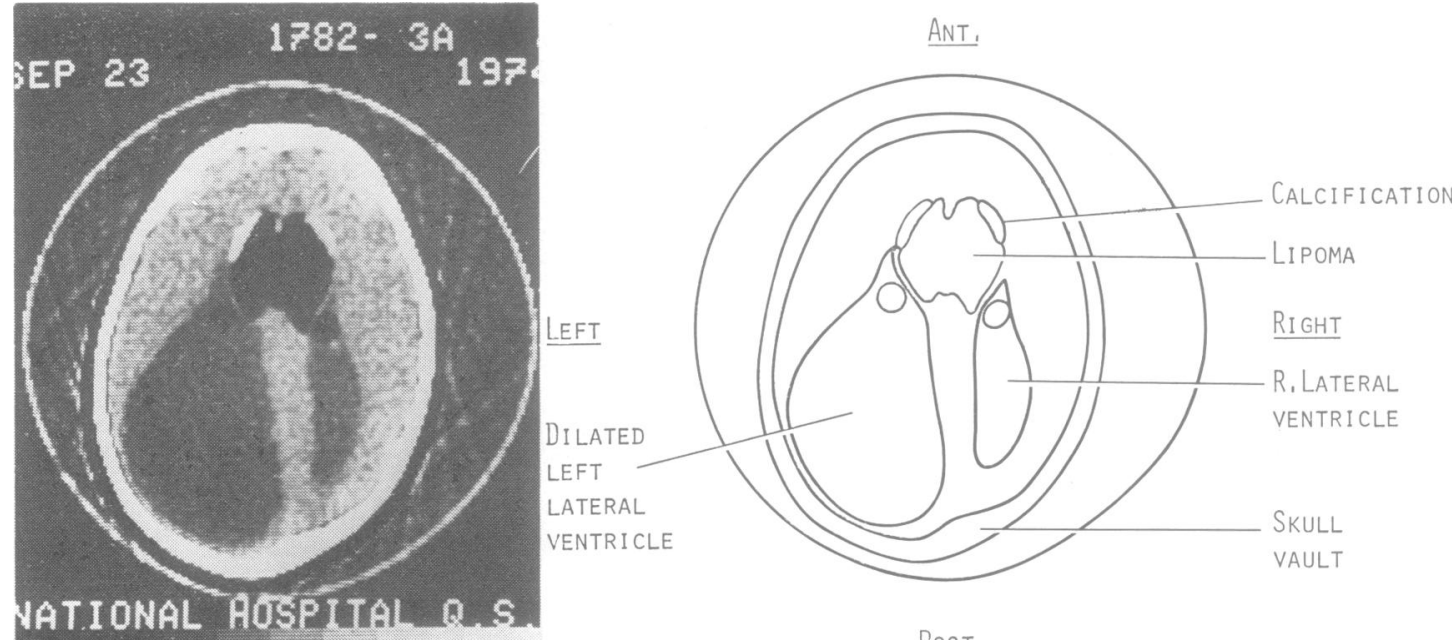

POST,

FIG. 3. Case 3. EMI scan showing the anteriorly placed lipoma of corpus callosum, calcification around its surface, hydrocephalus, and porencephaly of the left lateral ventricle. 
cause of the lipoma. They believed that the lipoma and the agenesis of the corpus callosum were produced by two distinct pathological processesnamely, meningeal maldifferentiation and dysraphism -and that these two processes were possibly genetically related.

\section{MACROSCOPIC APPEARANCES}

The size varies from small nodules, less than $1 \mathrm{~cm}$ diameter, to large masses. There is usually a dense, collagenous capsule, adherent to adjacent brain. The anterior cerebral arteries usually traverse the length of the tumour and do not deviate significantly from their normal courses. As in the two cases reported here, the anterior cerebral artery is often present as a single enlarged vessel traversing the tumour, and dividing into pericallosal and callosomarginal vessels (Danziger, 1972). The vessels may show considerable irregularity of calibre. The lipomas are often highly vascular. There may be an associated subcutaneous lipoma at the vertex, connected to the lipoma of the corpus callosum by a slender stalk which passes through a skull defect.

\section{MICROSCOPIC APPEARANCES}

Microscopically the lipoma consists of typical adipose tissue cells, with a variable amount of collagen, often obvious at points of contact with nervous tissue, which may penetrate brain tissue in association with blood vessels. Ganglion cells and glial elements may be present. Small accretions of calcium occur within the capsule of the lipoma, in the lipoma itself, or in the surrounding brain tissue. The presence of bone has also been described (Rubenstein, 1932). The curvilinear calcification is probably in the surrounding brain tissue (Patel, 1965). There are usually numerous large blood vessels coursing through the tumour.

\section{ASSOCIATED FEATURES}

The commonest associated anomaly is agenesis of the corpus callosum $(48 \%$ of cases according to Zettner and Netsky, 1960). The agenesis may affect part or all of the corpus callosum (Patel, 1965). There may be associated cerebral hemiatrophy, as present in both cases. Associated structural malformations of the anterior cerebral artery are described above, as are associated dysraphic disturbances. Other associated anomalies include webbed toes, cleft lip, mongolism, funnel chest, facial asymmetry, high arched palate, cardiac ventricular septal defect, agenesis of the cerebellar vermis (Zettner and Netsky, 1960), pituitary tumour, and acoustic neurofibroma (Patel, 1965). Associated intracranial lipomas, most commonly in the choroid plexus of the lateral ventricle, have been reported.
There may be hydrocephalus or porencephaly. There has been a recent report of the condition in association with muscular dystrophy (Jayam et al., 1969).

\section{PRESENTING FEATURES}

Approximately $50 \%$ of cases are asymptomatic. The commonest presentation is epilepsy, either grand mal or petit mal. Headache, vomiting, hemiplegia, and vertigo may occur. There may be mental retardation or emotional lability. The origin of the epilepsy in this condition is not clear. One theory advanced is that the invasion of cerebral cortex by the collagenous capsule sets up an irritative focus, producing epilepsy in the way that brain scarring supposedly produces post-traumatic epilepsy. The epilepsy does not seem to be related to local pressure, as there are no associated signs of pressure atrophy in nearby brain (Patel, 1965). Papilloedema is a rare occurrence, described in two previous cases (Nordin et al., 1955; Patel, 1965) in neither cases was its occurrence readily explained. Visual field defects were noted in case 2 above.

\section{RADIOLOGY (Fig. 1)}

The plain skull radiographic findings are diagnostic (Sosman, 1946). In typical cases there is a radiolucent zone in the frontal region, often comma shaped; this is surrounded by curvilinear calcification along its lateral margins. The lesion is usually symmetrically disposed with respect to the midline (Taveras and Wood, 1964). Unilateral calcification is sometimes seen (Craddock and Riddervold, 1972). While the calcification is usually in the form of linear fine speckling along the margins of the tumour, it may occasionally occur in large accretions of varying sizes, sometimes as large as $1.5 \mathrm{~cm}$ (Patel, 1965; Larsen and Stiris, 1970) and may lie in the central part of the lesion (Patel, 1965). Deviation of the crista galli with asymmetry in shape and thickness of the skull vault, may indicate underlying hemiatrophy of the brain.

Pneumoencephalography may show hydrocephaly, evidence of agenesis of the corpus callosum, symmetrical separation and distortion of the anterior horns and bodies of the lateral ventricles by the lipoma. There may be little visualisation at all of the anterior horns of the lateral ventricles; there may be dilatation of the foramina of Monro and the third ventricle. The third ventricle may be displaced by the lipoma or elevated in association with agenesis of the corpus callosum. A porencephalic cyst may be present.

Carotid angiography may show the anterior cerebral vessel or vessels, often enlarged, or the pericallosal vessels passing through the lesion. If the anterior cerebral artery is single it divides into 
pericallosal and callosomarginal vessels. There may be evidence of an avascular space-occupying lesion in the frontal region, or a fine pathological circulation may be seen. There may be angiographic evidence of hydrocephalus. The callosal vessels may be displaced from the midline when hemiatrophy is present.

The EMI scan will be diagnostic in this condition, as it may demonstrate that the density of the lesion corresponds to that of adipose tissue and that there is associated calcification. The EMI scan is most useful in defining associated anomalies such as hydrocephalus, porencephalic cyst, associated lipomas, and vault asymmetry.

\section{MANAGEMENT}

If the condition is detected accidentally there is no treatment. If seizures have occurred, anticonvulsants should be given because of the risk of recurrent epilepsy. Surgery has little place in the management of the condition. Surgical management of hydrocephalus could conceivably be necessary if pressure symptoms or progressive dementia occurred. Surgical removal of the lipoma is unattractive, and there is a dismal record of surgery in this group of patients. Zettner and Netsky (1960) reported the results of surgery in 16 cases, in which there were 10 deaths, and three cases in which the clinical state was worsened. In the six survivors, removal was incomplete in all but one. In only two cases was the recovery uneventful, and only one patient was improved by operation. Since that article was published, two further cases of postoperative death have been reported (Patel, 1965; Sukthomya and Menakanit, 1973). The close relationship of the anterior cerebral vessels to the lesion makes damage to these vessels a real operative hazard. In the case reported by Sukthomya and Menakanit, (1973) death was preceded by a state of akinetic mutism which followed the tying off of the anterior cerebral vessels. Firm adhesion between the collagenous capsule and brain together with the high vascularity of the tumour add to the surgical hazard. The necessity for removal of a lipoma of the corpus callosum must be seriously questioned, since it is a non-malignant, nonprogressive lesion, and some patients live to old age, trouble-free, untreated. Epilepsy, the commonest symptom produced by lipomas of the corpus callosum is unlikely to be relieved by surgery.

It is evident that the EMI scan is the least traumatic means now available of investigating the patient with a lipoma of the corpus callosum.

I thank Mr Kenneth Till and Mr Norman Grant (Neurosurgical Department of the Hospital for Sick Children, Great Ormond Street, London) for permission to report their cases; Dr J. Gawler (the National Hospital, Queen Square, London) for the EMI scanning reports, and Dr J. Bull (Director, Department of Radiology, National Hospital, Queen Square, London) for permission to report the EMI scan; Dr R. D. Hoare (Consultant Neuro-radiologist, Hospital for Sick Children, Great Ormond Street, London) for the radiological data.

\section{REFERENCES}

Arseni, C., and Maretsis, M. (1972). Lipoma of corpus callosum (in Rumanian). Neurologia, Psychiatria, Neurochirurgia, 17, 15-20.

Bromowicz, J. (1970). A case of lipoma of the corpus callosum (in Polish). Neurologia, Neurochirurgia Polska, 4, 739-743.

Cooper, W. C., and Von Hagen, K. O. (1962). Lipoma of corpus callosum. Bulletin of the Los Angeles Neurological Society, 27, 39-44.

Craddock, W. E., and Riddervold, H. O. (1972). Uni lateral calcification in a lipoma of the corpus callosum? Journal of the Canadian Association of Radiologists, 23 192-194.

Danziger, J. (1972). Agenesis of corpus callosum witt? azygos anterior cerebral artery, a lipoma, and porence $-\rightarrow$ phalic cyst. South African Medical Journal, 46, 739-7410

Jayam, A. V., Banerji, A. P., and Lalitha, V. S. (1969). A case of muscular dystrophy with probable lipoma of the corpus callosum. Neurology (Bombay), 17, 85-88.

Larsen, J. L., and Stiris, G. (1970). Lipoma of the corpus callosum with atypical calcification. British Journal of Radiology, 43, 576-577.

List, J. F., and Everett, M. (1946). Lipoma of the corpus callosum: a clinico-pathologic study. American Journal of Roentgenology, 55, 125-134.

Meyer, T. L., and Leimbach, W. (1970). Lipoma of the corpus callosum: report and review. Ohio Medical Journal, 66, 492-495.

Nordin, W. A., Tesluk, H., and Jones, R. K. (1955). Lipoma of the corpus callosum. Archives of Neurology and Psychiatry (Chic.), 74, 300-306.

Patel, A. N. (1965). Lipoma of the corpus callosum. A non-surgical entity. North Carolina Medical Journal, 26, 328-338.

Rokitansky, C. (1856). Lehrbuch der Pathologischen Anatomie, vol. 2, pp. 468. Braumueller: Vienna.

Rubenstein, B. G. (1932). Ueber einen Fall von Unvollstaendig Tehlendem und durch Fettge webe Ersetztem Balken. Frankfurter Zeitschrift für Pathologie, 44, 379.

Sosman, M. C. (1946). Discussion of papers by A. P. N Echternucht and J. A. Campbell. Midline anomalies of brain. Radiology, 46, 119. 
Sukthomya, C., and Menakanit, V. (1973). Lipoma of the nervous system. Report on 2 cases. Australasian Radiology, 17, 256-260.

Taveras, J. M., and Wood, E. H. (1964). Diagnostic Neuro-Radiology, 2nd edn, pp. 1-213. Williams Wilkins: Baltimore.
Vonderahe, A. R., and Niemer, W. T. (1944). Intracranial lipoma. A report of 4 cases. Journal of Neuropathology and Experimental Neurology, 3, 344-354.

Zettner, A. and Netsky, M. (1960). Lipoma of the corpus callosum. Journal of Neuropathology and Experimental Neurology, 19, 305-319. 